\title{
A Comparison between Radial Rakes of Sensors and Axial Arrays of Microphones for the Experimental Investigation of Tone Noise in LPTs
}

\author{
F. Taddei ${ }^{1}$ and M. De Lucia ${ }^{2}$ \\ University of Florence, Florence, Italy, 50139 \\ D. Torzo ${ }^{3}$ and E. Spano ${ }^{4}$ \\ Avio group S.p.A., Rivalta di Torino, Italy, 10040
}

\begin{abstract}
The experimental investigation of turbomachinery noise requires to measure the acoustic field within annular test rigs. For this purpose, different in-duct measurement techniques are available. The most widely used are the arrays of flush-mounted microphones installed at the duct walls and the rakes of dynamic pressure sensors placed along the radial direction of the duct. Both methods have been implemented and used for several test campaigns performed at the Avio cold flow rig in Turin (Italy), in order to assess the acoustic performances, from the tonal noise point of view, of a model low-pressure-turbine representative of modern turbofan applications. This paper aims at performing a back-toback comparison between the results obtained by using these different experimental techniques. The results allowed to make assessments about the effectiveness of each experimental technique at analyzing tonal noise, highlighting the peculiar features of the two methods and their relative advantages and drawbacks.
\end{abstract}

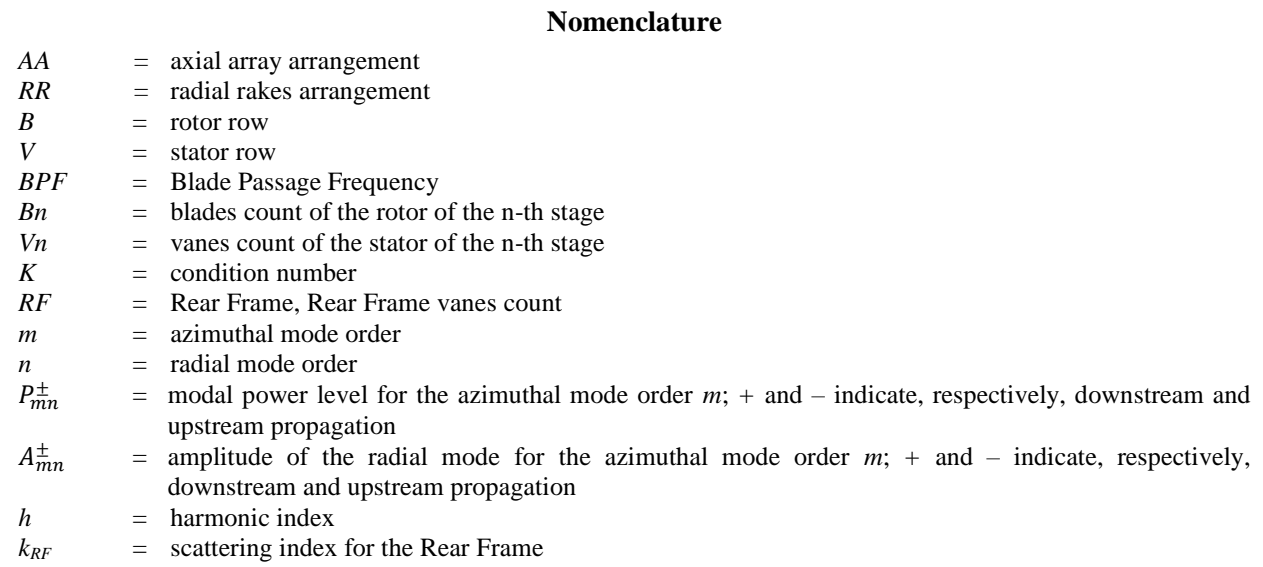

\footnotetext{
${ }^{1}$ Ph.D. research fellow, Energy Department S.Stecco, via S. Marta 3, 50139, Florence, Italy.

${ }^{2}$ Full professor, Energy Department S.Stecco, via S. Marta 3, 50139, Florence, Italy.

${ }^{3}$ Ph.D., R\&D, Avio group S.p.A., via $1^{\circ}$ maggio 56, Rivalta di Torino, 10040, Italy.

${ }^{4} \mathrm{R} \& \mathrm{D}$ lead engineer, Avio group S.p.A., via $1^{\circ}$ maggio 56, Rivalta di Torino, 10040, Italy.
} 